\title{
Real-time Measurement of the Single Nanoparticle Electrophoretic Mobility
}

\author{
Bohdan Yeroshenko ${ }^{1, *}$, Wouter Wassing ${ }^{1}$, Allard P. Mosk ${ }^{1,}$, and Sanli Faez ${ }^{1, * *}$ \\ ${ }^{1}$ Utrecht University, Debye Institute for Nanomaterials Science, Utrecht, 3584 CS, Netherlands
}

\begin{abstract}
The electrophoretic mobility of a single nanoparticle depends on its surface charge and its environment. Thus the change of the mobility can reflect the change in its chemical and physical properties. We present a high-bandwidth method to measure the electrophoretic mobility, based on optical tweezers and electrophoresis. We envision studying of nanoscale chemical processes as a possible application of this method.
\end{abstract}

\section{Introduction}

A wide range of processes is happening on the surfaces of nanoparticles in both industrial chemistry and biology, such as catalysis and enzymatic activity. These processes are associated with changes to the particle properties such as surface charge, polarizability and hydrodynamic properties. One way to study these changes is by studying the electrophoretic mobility; by confining the particle in space by optical tweezers and applying an external oscillating electric field. It was shown that single charge transfer is measurable with this method in a non-polar solvent [1]. In other reports, electrophoresis was studied on single particles using digital signal processing [1][2][3]. We extend those methods to the real time measurement of the mobility and thus extract the charge of a nanoparticle.

\section{Motion of a nanoparticle in an optical trap in an oscillating electric field}

Diffusion of a single nanoparticle in a liquid can be described as a random walk, if we consider time scales bigger than those associated with inertial effects, $t_{s}>m / \gamma_{0}$. Then the motion of particle can be described as a Brownian noise:

$$
S_{B N}=\frac{D / 2 \pi^{2}}{f^{2}}
$$

where $D$ is a diffusion constant and $f$ is frequency. The description of the forces acting on the nanoparticle in the optical tweezers vary depending on the size of the particle in relation to the wavelength of the trapping beam, but can be split into the optical scattering force, that pushes the particle in the direction of the light propagation, and the optical gradient force that pushes the particle in or out of the highest intensity of the light. The force can be accurately approximated as a harmonic potential. The power spectrum of the motion will then be:

$$
S_{O T}=\frac{D / 2 \pi^{2}}{f^{2}-f_{c}^{2}}
$$

where $f_{c}$ is a corner frequency. This means that the optical trap effectively attenuates the low frequency motion, at frequencies smaller than the corner frequency of the trap, and does not affect the motion at higher frequencies. The fast displacements can thus be considered the same as that of a freely diffusing nanoparticle. When the external field is applied to a particle in a solvent, it will start to move in the field with a proportional drift velocity. The drift velocity is related to the external field and the electrophoretic mobility as

$$
v_{e p}=\mu E .
$$

where $\mu$ is an electrophoretic mobility and $E$ is magnitude of the external electric field. The electrophoretic mobility depends on properties of the media the nanoparticle is in, such as its viscosity and dielectric constant, and the properties of the particle, its size and most importantly, for this application, the zeta potential. Zeta potential, $\zeta$, is the electric potential at the slip plane, a plane in the electric double layer around the particle, that denotes a first plane from the surface of the particle, where the ions are mobile [4].

$$
\mu=\frac{\epsilon_{0} \epsilon_{r}}{\gamma} \zeta
$$

By measuring the amplitude of the oscillation in space at that frequency, we can immediately measure its mobility and thus zeta potential and thus we can calculate the charge state of the particle. In the case when the applied voltage is harmonic, and the period of the applied voltage longer than the relaxation time of the charges around the particle, the motion at the applied frequency can be described as:

$$
S_{E P}=\frac{\left|E^{2}\right|}{f^{2}} \delta\left(f_{\text {ext }}\right) *|\mathcal{F}\{\mu(t)\}|^{2}
$$

where $f_{\text {ext }}$ is a frequency of the applied field and $\mathcal{F}\{\mu(t)\}$ is a Fourier Transform of the mobility change in time. Thus the change of the mobility will be present in the power spectrum of the motion of the particle as a broadening of the peak around the applied frequency, see Fig.1.

\footnotetext{
*e-mail: b.yeroshenko@uu.nl

**e-mail: s.faez@uu.nl
} 

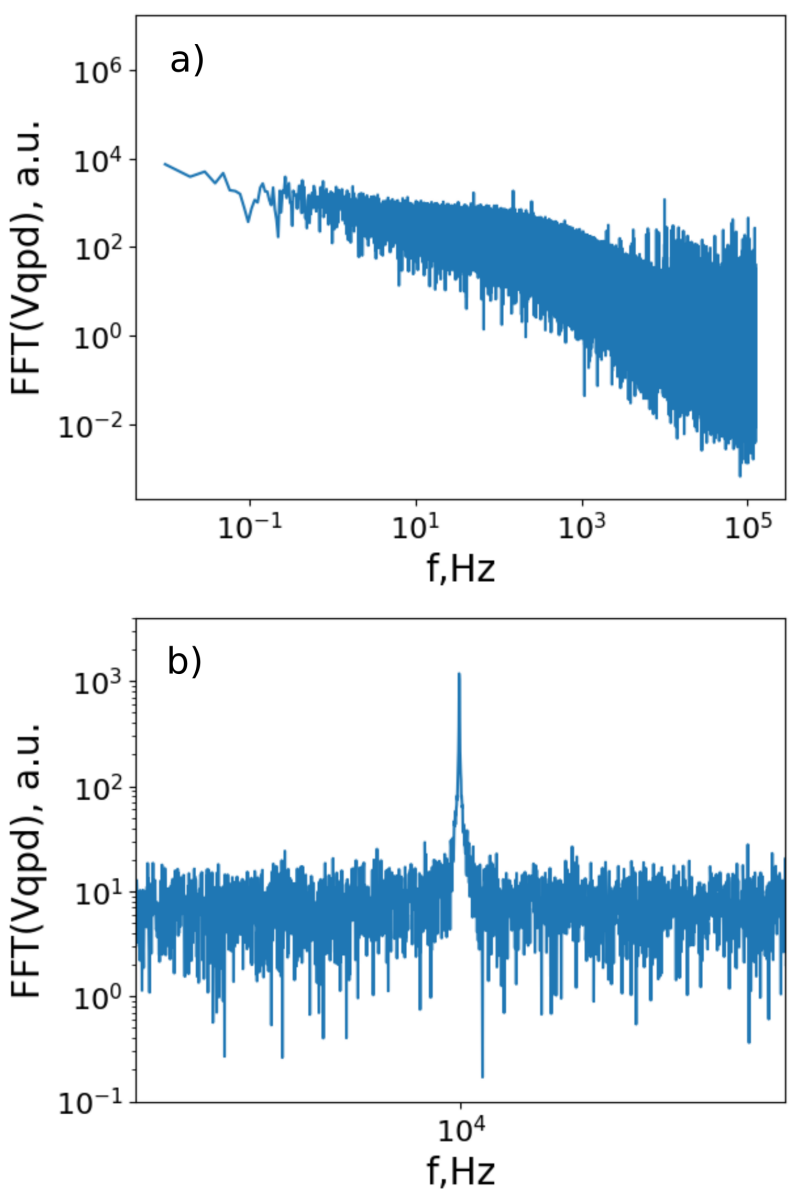

Figure 1. Fast Fourier Transform of the voltage readings of the quadrant photodiode (QPD) corresponding to the motion of the $490 \mathrm{~nm} \mathrm{TiO}_{2}$ nanoparticle inside of the optical tweezer a) full spectrum b) around the frequency of the applied field with characteristic broadening of the peak

Thus by using a lock-in amplifier one can directly measure changes to the electrophoretic mobility. Time resolution of this measurement is limited only by the low pass filter properties of the lock-in amplifier. The noise of the signal is the Brownian noise of the particle around the applied frequency. And the signal to noise ration (SNR) can be increased by applying a larger electric field, up to a certain limit dictated by the chemical stability of the solution.

\section{Methods}

A $1064 \mathrm{~nm}$ laser is used for trapping, with usual power of the beam being around $300 \mathrm{~mW}$. Detection is done by both a camera and a quadrant photodiode (QPD). The QPD allows fast signal sampling, with maximum at around 700
$\mathrm{kHz}$. It allows to continuously monitor the total reflected signal, which in turn allows detection of a trapping event and the relative position of the particle at the same time. We use a polarized beam splitter and a quarter-wave plate for maximization of signal collection. We apply the electric field through microfabricated electrodes in the microfluidic cell. The signal at the actuation frequency is separated using a lock-in amplifier.

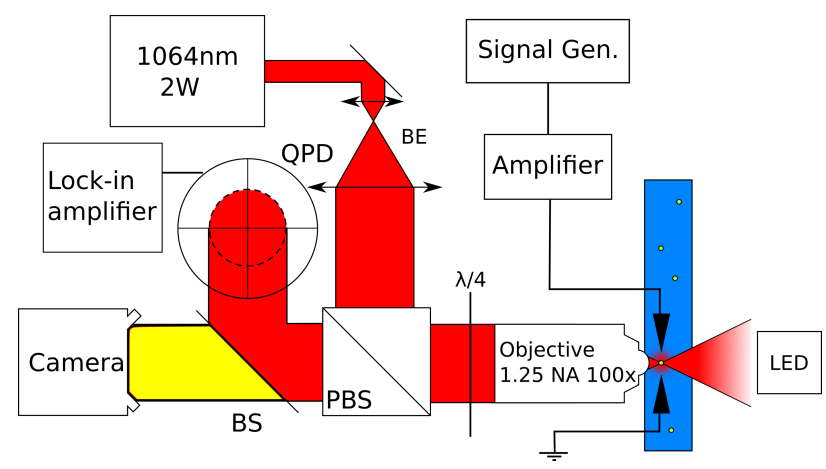

Figure 2. Schematic of the optical tweezer setup in the backscattering configuration

\section{Discussion}

Our experiment can measure the electrophoretic mobility in real time with time resolution of around $1 \mathrm{~ms}$ and is limited in duration by the trapping time which can be hours. This setup is able to provide both the precise measurement of the mobility of the particle, its change due to fluctuation of the charges in the electric double layer, and its change under external influence. With careful experimental design we expect that we will be able to measure polarizability, Stern potential and Zeta potential of a single nanoparticle. [4].

This research is supported by the Dutch Science Foundation (NWO).

\section{References}

[1] F. Beunis, Strubbe, F., K. Neyts, D. Petrov, Phys. Rev. Lett. 108 p.016101 (2012)

[2] O. Otto, C. Gutsche, F. Kremer, U. Keyser, Rev. Sci. Instrum. 79 p.023710 (2008)

[3] G.S. Roberts, T.A. Wood, W. Frith, P. Bartlett, J. Chem. Phys. 126 p.194503. (2007)

[4] S.S. Dukhin, B.W. Derjagin, Electrophoresis (Nauka, Moscow, 1976), pp. 74, 146 